\title{
Electron and Heat Transport in Porphyrin-based Single- Molecule Transistors with Electro-burnt Graphene Electrodes
}

\author{
Hatef Sadeghi, ${ }^{\star *}$ Sara Sangtarash and Colin J Lambert ${ }^{1 \dagger}$ \\ ${ }^{1}$ Quantum Technology Centre, Physics Department, Lancaster University, Lancaster, LA1 4YB, UK \\ *h.sadeghi@lancaster.ac.uk, ${ }^{\dagger}$.lambert@lancaster.ac.uk
}

\begin{abstract}
We have studied the charge and thermal transport properties of a porphyrin-based single-molecule transistor with electro-burnt graphene electrodes (EBG) using the non-equilibrium Green's function method and density functional theory. The porphyrin-based molecule is bound to EBG electrodes by planar aromatic anchor groups. Due to efficient pi-pi overlap between the anchor groups and graphene and the location of frontier orbitals relative to the EBG Fermi energy, we predict HOMO-dominated transport. An on-off ratio as high as $\mathbf{1 5 0}$ is predicted for the device which could be utilized with small gate voltages in the range of $\pm 0.1 \mathrm{~V}$. A positive thermopower of $280 \mu \mathrm{V} / \mathrm{K}$ is predicted for the device at the theoretical Fermi energy. The sign of the thermopower could be changed by tuning the Fermi energy. By gating the junction and changing the Fermi energy by $+10 \mathrm{meV}$, this can be further enhanced to $475 \mu \mathrm{V} / \mathrm{K}$. Although the electrodes and molecule are symmetric, the junction itself can be asymmetric, due to different binding configurations at the electrodes. This can lead to rectification in the current-voltage characteristic of the junction.
\end{abstract}

Single-molecule transistor | electro-burnt graphene electrodes | nanoelectronics | porphyrin

$\mathbf{T}$ ransistors are the fundamental building block of modern electronic devices used to amplify or switch electronic signals. Most common transistors contain three terminals, two of which carry a current from the source to the drain and the third (gate or base) controls the current through the transport channel to either amplify the input current in bipolar junction transistors (BJT) or switch the voltage in field effect transistors (FET) [1]. Recently the idea of two terminal molecular-scale transistors has been proposed in which a molecule forms the conducting channel of the transistor [2].

To sustain Moore's law [3] the number of transistors in an integrated circuit needs to double approximately every two years. Since 1974, when the idea of a single molecule transistor was proposed by Ratner and Aviram [4], many attempts to realize FETs have failed to deliver the room-temperature reliability, reproducibility and stability required by the electronics industry $[5,6]$. Transport in singlemolecule FETs is mostly dominated by the contacts between the electrodes and molecules which is an obstacle to achieving reproducibility and stability. Furthermore in laboratory-based conductance measurements, using for example mechanically-controlled break junctions, gold is widely employed as the electrode material, to avoid oxidation and degradation of electrodes and because of its high atomic mobility. However, gold nano-electrodes are unstable at room temperature [5].

Recently an alternative strategy for the fabrication of the stable electrodes with the nanometre separation has been proposed using the sp2-bonded two-dimensional carbon-based material, graphene [7, 8]. Graphene nano-junctions are attractive as electrodes for electrical contact to single molecules [8-14], due to their excellent stability and conductivity up to high temperatures and a close match between their Fermi energy and the HOMO (highest occupied molecular orbital) or LUMO (lowest unoccupied molecular orbital) energy levels of organic materials. Graphene electrodes also facilitate electrostatic gating due to their reduced screening compared with more bulky metallic electrodes.

Here, we study charge and thermal transport through a porphyrin single-molecule transistor with electro-burnt graphene electrodes using the non-equilibrium Green's function method and density functional theory. First we discuss the electronic structure of the prophyrin molecule. Then we study the electro-burnt graphene electrodes and finally the two-terminal device in which the anchor groups of the porphyrin molecule bind to the graphene electrodes by pi-orbital overlap. We then discuss the contribution of each part of the molecule to charge transport by the means of an effective tight binding model. Finally we investigate the thermoelectric properties of the device.

\section{A Porphyrin SET Molecular Structure}

Figure 1 shows the porphyrin FET molecular structure, which consists of two electro-burnt graphene electrodes connected to drain and source reservoirs ( $\mathrm{D}$ and $\mathrm{S}$ ) and the porphyrin molecule (PM). The molecule consists of two "butterfly" anchor groups connected to the porphyrin core via a carbon acetylene spacer as shown in fig. 1a. The anchor groups bind to the surface of the graphene through pi-pi interactions. The central porphyrin is also connected to two side groups, which stabilize the molecule within the junction. 


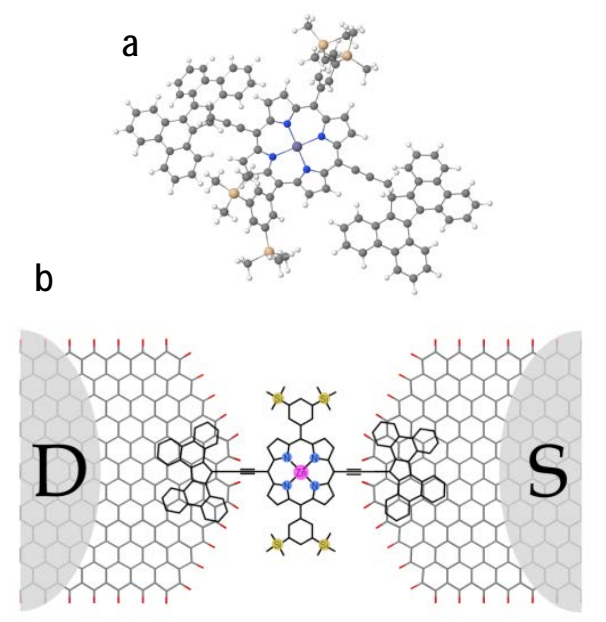

Figure 1: Porphyrin SET Molecular Structure. a) porphyrin molecule with butterfly backbone, b) the device structure consist of two electro-burnt graphene electrodes, a porphyrin molecule and reservoirs $D$ and $S$. The butterfly anchor groups of the porphyrin molecule are laid on the surface of the graphene through $p-p$ interaction to make mixed $A A$ and $A B$ stacking with graphene.

We first use DFT to study the electronic structure of the PM. To characterize the gas phase molecule, the isolated PM shown in fig. $1 \mathrm{a}$ is relaxed to reach the ground state energy (see methods). We carried out a spin-polarized calculation since the $d$ orbitals of the $\mathrm{Zn}$ atom could be filled to different degrees. It is well-known that Kohn-sham DFT eigenvalues usually underestimate the HOMOLUMO gap and DFT typically does not predict their correct location relative to the Fermi energy of the electrodes. However, the LUMO (HOMO) energy level is almost equivalent to the negative of the electron affinity energy (ionization potential). Therefore, we estimate the gap $E_{g}$ of the molecule (sometimes called additional energy) by computing the electron affinity $(E A)$ and ionization potential $(I P): \quad E_{g}=I P-E A$. EA and IP are calculated from the total energies of the neutral and N \pm -electron states of the molecule: $I P=E(N-1)-E(N)$, and $E A=E(N)-E(N+1)$. For the PM, the IP and $E A$ are calculated as $-1.36 \mathrm{eV}$ and $-5.2 \mathrm{eV}$ respectively which yields $E_{g}=3.84 \mathrm{eV}$. The Kohn-sham DFT eigenvalues predicts the $E_{H O M O-1}^{D F T}=-4.46 \mathrm{eV}, E_{H O M O}^{D F T}=-4.37 \mathrm{eV}$, $E_{L U M O}^{D F T}=-2.85 \mathrm{eV}$ and $E_{L U M O+1}^{D F T}=-2.62 \mathrm{eV}$ using the GGA-PBE functional which yields the Kohn-sham DFT gap as: $E_{g}^{D F T}=$ $E_{L U M O}^{D F T}-E_{H O M O}^{D F T}=1.52 \mathrm{eV}$ for the gas phase molecule.
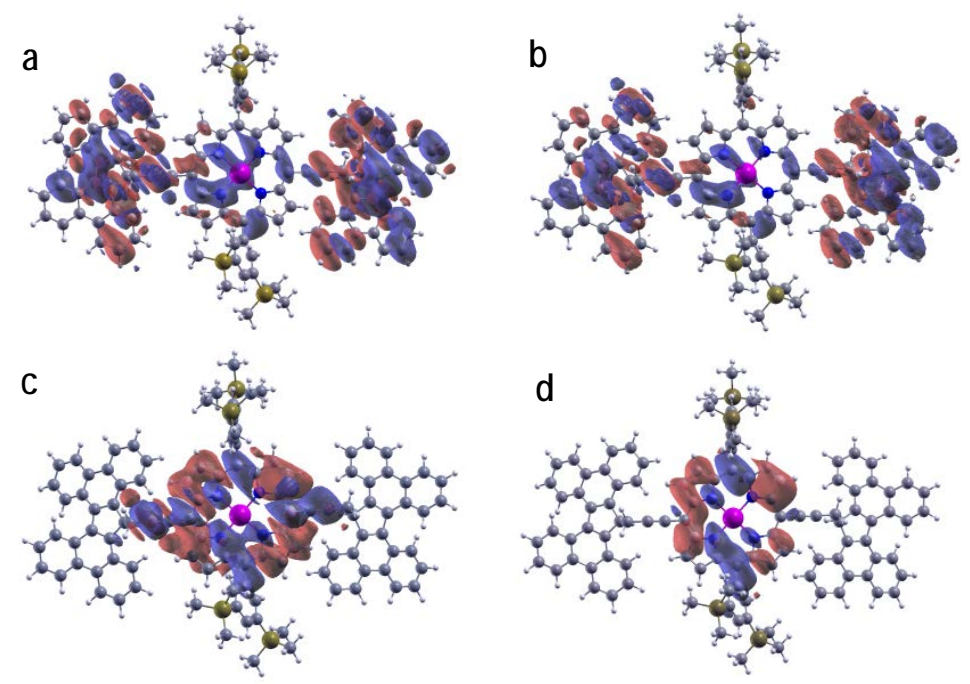

Figure 2: a) HOMO-1, b) HOMO, c) LUMO and d) LUMO+1 state iso-surfaces

Figure 2 shows the HOMO-1, HOMO, LUMO and LUMO+1states iso-surfaces. The wave function of the HOMO-1 and HOMO states are mostly are delocalized over the PM and the butterfly anchors, whereas the LUMO and LUMO+1 states are localized on the porphyrin central group. This suggests transport should be HOMO dominated. However, the molecular orbitals are localized in the porphyrin central group in the LUMO and LUMO+1 state.

\section{Electro-burnt graphene electrodes}

Feedback-controlled electro-burned graphene (EBG) electrodes with nanometre separation have been formed in mechanical exfoliated few-layer graphene [7] and CVD grown monolayer graphene [15, 16]. To form the EBG electrodes, first a sufficiently high bias voltage is 
applied to crack the ribbon. This usually happens in the centre of the ribbon due to higher temperature induced in this region [17]. This high temperature in the constricted part of the graphene nanoribbon causes carbon atoms to react chemically with atmospheric oxygen atoms and burn. A feedback-signal is used to stop burning before destroying the sample. After successive repetitions of this process, the graphene nanoribbon becomes more and more narrow and finally breaks to create a nanometre size gap. These electrodes can then trap a molecule in the gap and be used to study its electrical properties. Moreover, the ability to place a gate electrode beneath the gap makes this an excellent platform for tuning and studying quantum effects in single-molecule transport.

a
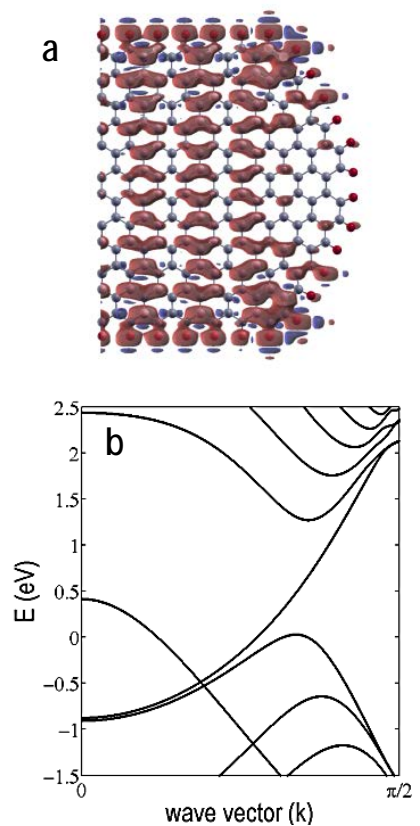
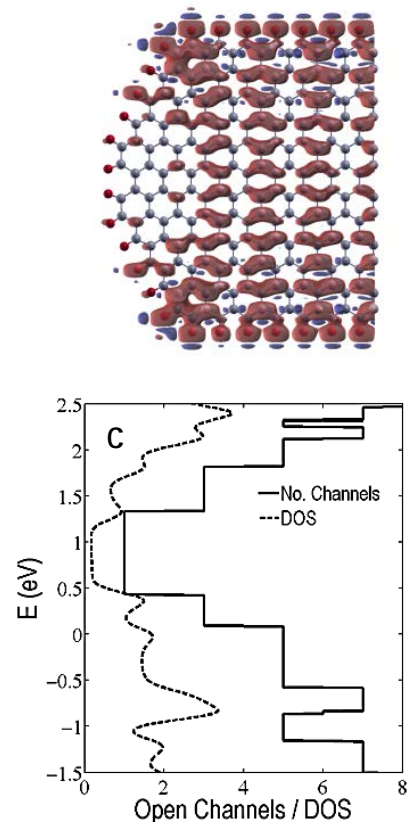

Figure 3: molecular and electronic structure of the EBG electrodes. a) molecular orbital levels, b) band structure of EBG electrodes, c) density of states and number of open channels in the EBG electrodes

Due to the burning process, the edges of the EBG most probably are terminated by oxygen specifically close to the junction. So, before studying the transport properties of the PM, we focus on the transport properties of the EBG electrodes with oxygen-terminated edges. Figure 3 shows the molecular and electronic structure of the EBG electrode. The electrode is a $3 \mathrm{~nm}$ wide zigzag semi-infinite graphene nanoribbon terminated by oxygen connected to a half-ellipse like structure as shown in fig. $3 \mathrm{a}$. The molecular orbital levels in the Fermi energy $E_{F}=0 \mathrm{eV}$, indicates that the orbitals are mostly localized in the edges of the ribbon. It is apparent that the up-spin is mostly located toward the edges in contrast with the down spins, which are delocalized over the EBG. The band structure of the electrode is bent in the vicinity of the $\mathrm{k}$ point due to the edge states associated with the oxygen atoms (fig. 3b). Due to the high electronegativity of oxygen atoms, charge is transferred from the carbon atoms to the oxygen atoms. Consequently, oxygen-terminated ribbons show p-type doped behaviour $[18,19]$ and their density of states (DOS) is shifted toward positive energies as shown in fig. $3 b$ (dashed line). Figure $3 \mathrm{~b}$ (solid line) also shows the number of open conduction channels in ideal oxygen terminated EBG electrodes. Due to the p-type behaviour of EBG electrodes, the open channels are shifted toward the positive energies.

\section{Electron and thermal transport in EBG-PM junction}

We now consider transport through the molecule inside the nanogap. In the equilibrium configuration, the PM butterfly anchor groups assume a mixed $A A$ and $A B$ stacking with the graphene surface. Figure 4 shows the resulting transmission probability for the electrons of energy $E$ passing from the left to the right electrode at zero and room temperature, obtained from the DFT mean-field Hamiltonian of the system combined with our Green's function (GF) code (see methods). Due to the presence of oxygen atoms in the edges of the EBG electrodes, the Fermi energy is predicted to shift by about $0.45 \mathrm{eV}$, compared with hydrogen terminated electrodes and bare EBG electrodes. The HOMO-1, HOMO and LUMO resonances are labelled in fig. 4. As discussed above, the DFT predicted Kohn-Sham eigenvalues for the HOMO and HOMO-1 states are quite close to each other, which is why the HOMO and HOMO-1 resonances overlap. Moreover, the Fermi energy is close to the HOMO resonance indicating HOMO dominated transport, consistent with the local density calculation shown in fig. 2 for gas phase molecule. The inset of figure 4 shows the zero-bias current of the PM-EBG device at zero and room temperature. 


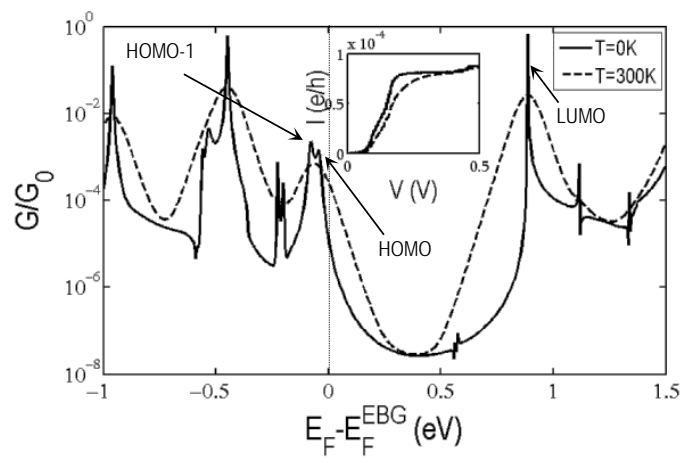

Figure 4: Conductance $G / G_{0}$ as a function of the Fermi energy $E_{F}$ of the $E B G$ electrodes shown in fig. $1 \mathrm{~b}$.

Under non-equilibrium conditions at finite voltages, the transmission coefficient $T(E, V)$ depends on both electron energy $E$ and bias voltage $V$, which in the presence of asymmetries can lead to rectification in the PM-EBG device. To demonstrate this, we have calculated the I-V characteristic of the device using the voltage dependent transmission coefficient as shown fig. 5 for between [-1,1] $\mathrm{V}$ which is experimentally applicable voltage for this device before blown-up.

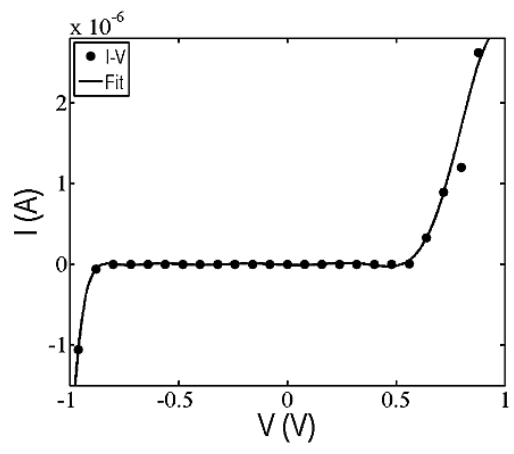

Figure 5: non-equilibrium I-V characteristic of the PM-EBG device

Remarkably the current is not symmetric relative to zero-bias point, even though the molecule itself and the leads are ideally symmetric. The asymmetry is due to differences in the angle between the PM butterfly anchor and the spacer between the prophryin and anchor, which causes the butterfly anchor makes slightly different configuration in the left and right electrodes. As a consequence, the junction configuration and coupling to the electrodes are slightly asymmetric, leading to rectification. Moreover, in a wide bias window $[-0.9,0.6]$ volts, the current is almost zero, but then suddenly increase with high current flow capability in the range of the $\mu \mathrm{A}$.

a
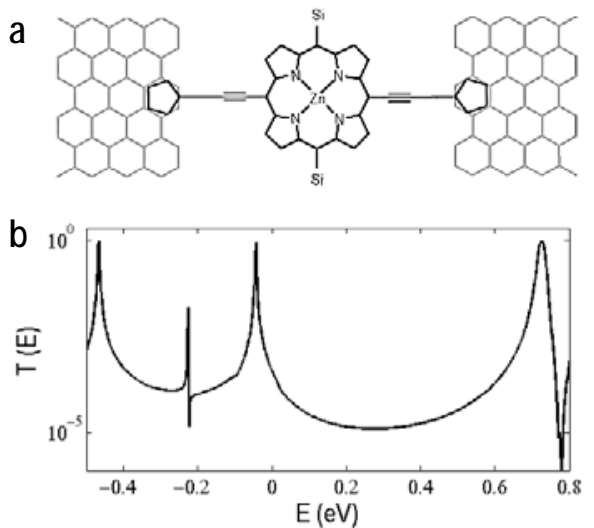
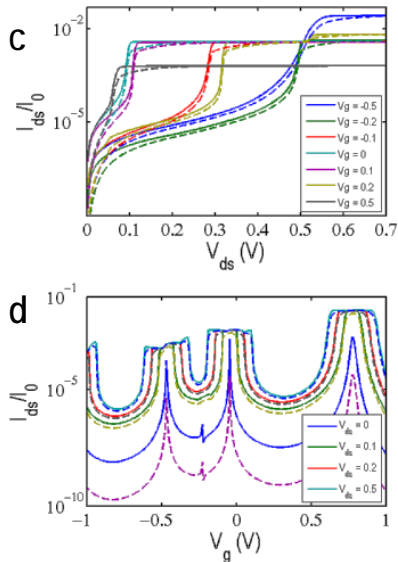

Figure 6: Tight-binding model of the PM-EBG device. (a) Schematic of the simplified device for tight-binding calculation, (b) transmission coefficient $T(E)$ using Hückel parameters, (c) drain source current $\left(I_{d s}\right)$ as a function of the drain source (bias) voltage $\left(V_{d s}\right)$ and $(d)$ drain source current as a function of the gate voltage $\left(V_{g}\right)$.

To further investigate transport through the device, we constructed a tight-binding model of the simplified device (figure 6a) using modified-Hückel parameters (see methods). The model is in good agreement with the $T(E)$ calculated from the DFT mean-field Hamiltonian as shown in $6 \mathrm{~b}$. A Fano resonance appearing close to the $E=-0.2 \mathrm{eV}$, is associated with the nitrogen atoms. By changing the coupling of the nitrogen-carbon they move and could create interesting features close to the HOMO which could be used in thermoelectric devices. Furthermore they could be split, if slightly asymmetric coupling between carbon and nitrogen applied. The zinc 
does not have effect on the electron transport as well as side groups (represented in figure 6a by the symbol "Si") since they have high orbital energies far ofromthe HOMO-LUMO resonances. Although the current mostly pass through the edge of the ribbon, they do not have much effect on the transport due to the weak coupling between the anchor and electrodes surfaces.

We used the TB model to investigate the behaviour of the PM-EBG device (fig. 1b) if a perpendicular gate voltage applied. This is modelled by calculating the gate voltage dependence of the transmission coefficient $T\left(E, V_{g}\right)$. To obtain a $V_{g}$-dependent $T B$ Hamiltonian, a gate potential is added to the diagonal terms of the TB Hamiltonian. Obviously this does not take the coulomb energy effect into account. Figure $6 \mathrm{c}$ shows the drain-source current $\left(l_{d s}\right)$ as a function of the drain-source (bias) voltage $\left(V_{d s}\right)$ at zero (solid lines) and room (dashed lines) temperature. When $V_{g}=0.5 \mathrm{~V}$ is applied the $I_{d s}$ switches to on-state much faster and saturates than when $V_{g}=0$ or $-0.5 \mathrm{~V}$ as shown in fig. $6 \mathrm{c}$. The on-off state of the device does not change monotonically by the gate voltage which could be explained by the asymmetry of the transmission coefficient around theoretical Fermi energy $(E=0)$. However, in the $[-0.1,0.1] \mathrm{V}$ interval, the current could be changed from $\sim 2 \times 10^{-5}$ to the $\sim 3 \times 10^{-3}$ which gives the ratio of the 150 . High variation of the current in small gate voltage window shows the potential of the device to perform in digital electronics. Figure $6 d$ shows the drain source current $\left(l_{d s}\right)$ as a function of the gate voltage $\left(V_{g}\right)$ in different bias voltages. When the bias voltage is very small $\left(V_{d s} \sim 0\right)$, the $I_{d s}-V_{g}$ dependence is quite similar to the $T(E)$. As expected, at higher bias voltages exceeding the width of the transmission resonance, $t / d$ s remains constant over a range of $V_{g}$ of order $V_{d s}$.
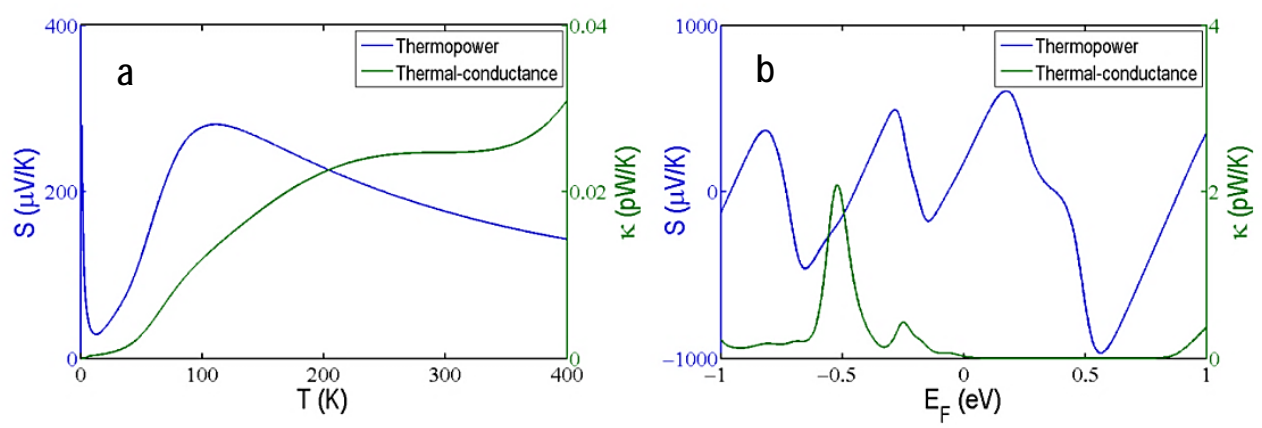

Figure 7: Thermal properties of the PM-EBG device. Thermopower (blue) and thermal conductance (green) as a function of the (a) temperature at $E_{F}=0 \mathrm{eV}$ and (b) Fermi energy $E_{F}$ at room temperature.

Figure 7 shows the thermopower $S$ and electronic contribution to the thermal conductance $\mathrm{k}$ of the PM-EBG device. The device could generate the maximum thermopower of $280 \mu \mathrm{V} / \mathrm{K}$ at $110 \mathrm{~K}$, which then decreases at higher temperatures as shown in figure $7 \mathrm{a}$. Furthermore, the thermal conductance of the device increases with temperature to the values of order $0.025 \mathrm{pW} / \mathrm{K}$ at room temperature. In addition, as shown in figure $7 \mathrm{~b}$, the thermopower changes sign for different Fermi energies $\left(E_{F}\right)$, because the Fermi energy is located close to a resonance. Consequently a variation in the Fermi energy as small as $10 \mathrm{meV}$, enhances the thermopower $475 \mu \mathrm{V} / \mathrm{K}$. In contrast the electronic thermal conductance of the device is quite low and does not change significantly by the small variation of the Fermi energy. The thermoelectric figure of merit could be high in this device provided the phonon contribution to the thermal conductance was small in comparable with the electronic contribution. Therefore, PM-EBG device shows great potential as a thermoelectric device.

\section{Conclusion}

We have investigated the electrical and thermoelectrical properties of a porphyrin-based molecule bound to the electroburnt graphene electrodes by planar aromatic anchor groups. Due to efficient pi-pi overlap between the anchor groups and graphene and the location of frontier orbitals relative to the graphene Fermi energy, we predict HOMO-dominated transport and a positive thermopower as high as $280 \mu \mathrm{V} / \mathrm{K}$. By gating the junction and tuning the Fermi energy, this can be further enhanced. Although the electrodes and molecule are symmetric, the junction itself can be asymmetric, due to different binding configurations at the electrodes. This can lead to slight rectification in the current-voltage characteristic of the junction.

\section{Computational Methods}

The Hamiltonian of the structures described in this paper were obtained using density functional theory (DFT) as described below or constructed from a simple tight-binding model (TB) as shown in figure 6 a. With a single orbital per atom of site energies: $\varepsilon_{C}=-4.05, \varepsilon_{H}=-20.5, \varepsilon_{S i}=-6.03, \varepsilon_{N}=-4.66, \varepsilon_{Z n}=-20.7$, and nearest neighbour couplings $\gamma_{C-C}=-2.7, \gamma_{C-H}=-0.23, \gamma_{C-N}=-4.2$, $\gamma_{C-S i}=-3.51$ and $\gamma_{N-Z n}=-2.9$ and couplings to the right and left electrodes $\gamma_{R S}=-0.27$ and $\gamma_{L S}=-0.35$, respectively.
DFT calculation: The optimized geometry and ground state Hamiltonian and overlap matrix elements of each structure was self-consistently obtained using the SIESTA [20] implementation of density functional theory. SIESTA employs normconserving pseudo-potentials to account for the core electrons and linear combinations of atomic orbitals to construct the valence states. The generalized gradient approximation (GGA) of the exchange and correlation functional is used with the Perdew-Burke-Ernzerhof parameterization (PBE) [21] a double- $\zeta$ polarized (DZP) basis set, a real-space grid defined with an equivalent energy cut-off of 150 Ry. The geometry optimization for each structure is performed to the forces smaller than 200 meV/Ang. The local density of state calculation has performed with 
polarized and in zero electronic temperature. For the band structure calculation, the EBG electrode was sampled by a 1x1x500 Monkhorst-Pack k-point grid.

Transport calculation: The mean-field Hamiltonian obtained from the converged DFT calculation or a simple tight-binding Hamiltonian was combined with our implementation of the non-equilibrium Green's function method, the GOLLUM [22], to calculate the phase-coherent, elastic scattering properties of the each system consist of left (source) and right (drain) electrodes and the scattering region. The transmission coefficient $T(E)$ for electrons of energy $E$ (passing from the source to the drain) is calculated via the relation:

$$
T(E)=\operatorname{Trace}\left(\Gamma_{R}(E) G^{R}(E) \Gamma_{L}(E) G^{R \dagger}(E)\right)
$$

In this expression, $\Gamma_{L, R}(E)=i\left(\sum_{L, R}(E)-\sum_{L, R}{ }^{\dagger}(E)\right)$ describe the level broadening due to the coupling between left $(L)$ and right $(R)$ electrodes and the central scattering region (S). $\sum_{L, R}(E)=H_{L S, R S}^{\dagger} G_{L, R} H_{L S, R S}$ are the retarded selfenergies associated with this coupling. $H_{L S, R S}$ and $G_{L, R}$ are the coupling matrix between $L S$ and $R S$ and the surface Green's function of the electrodes, respectively. $G^{R}=\left(E S-H_{S}-\sum_{L}-\sum_{R}\right)^{-1}$ is the retarded Green's function, where $H_{S}$ is the Hamiltonian of the scattering region and $S$ is overlap matrix.

Electrical properties: Using obtained transmission coefficient $(T(E))$, the conductance could be calculated by Landauer formula $\left(G=G_{0} \int d E T(E)(-\partial f\right.$ ) $\partial E$ )) where $G_{0}=2 e^{2} / h$ is the conductance quantum. In addition, the zero-bias current through the device at voltage $V$ could be calculated as:

$$
I(V)=\frac{2 e}{h} \int_{-\frac{V}{2}}^{+\frac{V}{2}} d E T\left(E, V_{g}\right)\left(f\left(E-\frac{V}{2}\right)-f\left(E+\frac{V}{2}\right)\right)
$$

where $f(E)=\left(1+\exp \left(\left(E-E_{F}\right) / k_{B} T\right)\right)^{-1}$ is the Fermi-Dirac distribution function, $T$ is the temperature, $V_{g}$ is gate voltage and $k_{B}=8.6 \times 10^{-5} \mathrm{eV} / \mathrm{K}$ is Boltzmann's constant. In non-equilibrium condition, the $I_{d s}-V_{d s}$ characteristic could be calculated from voltage dependent transmission $T\left(E, V_{d s}\right)$ as:

$$
I(V)=\frac{2 e}{h} \int_{-\infty}^{+\infty} d E T\left(E, V_{d s}\right)\left(f_{S}(E)-f_{D}(E)\right)
$$

where the $f_{S, D}$ are the electrochemical potential in the drain and source.

Thermal properties: The thermal properties of given device could be calculated from the transmission coefficient $(T(E))$. The thermopower $(S)$ and electronic contribution in thermal conductance $(\kappa)$ as a function of the temperature $(T)$ could be written as:

$$
\begin{gathered}
S(T)=-\frac{L_{1}}{e T L_{0}} \\
\kappa_{e}(T)=\frac{1}{h T}\left(L_{2}-\frac{L_{1}^{2}}{L_{0}}\right)
\end{gathered}
$$

where $L_{n}(T)=G_{0} \int d E\left(E-E_{F}\right)^{n} T(E)(-\partial f / \partial E)$.

\section{Acknowledgment}

This work is supported by UK EPSRC grants EP/K001507/1, EP/J014753/1, EP/H035818/1 and the European Union Marie-Curie Network MOLESCO.

\section{References}

1. Neamen DA (2003) Semiconductor physics and devices: basic principles (McGraw-Hill Science/Engineering/Math) 3 Ed

2. Tsutsui M \& Taniguchi M (2012) Single molecule electronics and devices. Sensors 12(6):7259-7298.

3. Moore GE (1965) Cramming more components onto integrated circuits. Electronics Magazine:4.

4. Ratner M \& Aviram A (1974) Molecular rectifiers. Chem. Phys. Lett 29:277-283.

5. Ratner M (2013) A brief history of molecular electronics. Nat. Nano. 8(6):378-381.

6. Perrin ML, Burzuri E, \& van der Zant HSJ (2015) Single-molecule transistors. Chemical Society Reviews.

7. Prins F, et al. (2011) Room-Temperature Gating of Molecular Junctions Using Few-Layer Graphene Nanogap Electrodes. Nano Lett. 11(11):46074611.

8. Burzurí E, Prins F, \& van der Zant HS (2012) Characterization of Nanometer-Spaced Few-Layer Graphene Electrodes. Graphene 1:26

9. Deng X, Zhang Z, Tang G, Fan Z, \& Yang C (2014) Spin filtering and large magnetoresistance behaviors in carbon chain-zigzag graphene nanoribbon nanojunctions. Phys. Lett. A 378(21):1540-1547.

10. Zanolli Z, Onida G, \& Charlier J-C (2010) Quantum spin transport in carbon chains. Acs. Nano. 4(9):5174-5180.

11. Akdim B \& Pachter R (2011) Switching behavior of carbon chains bridging graphene nanoribbons: effects of uniaxial strain. Acs. Nano. 5(3):17691774

12. Shen L, et al. (2010) Electron Transport Properties of Atomic Carbon Nanowires between Graphene Electrodes. J. Am. Chem. Soc. 132(33):1148111486

13. Katkov VL \& Osipov VA (2014) Planar graphene tunnel field-effect transistor. Appl. Phys. Lett. 104(5):053102.

14. Joachim C \& Ratner MA (2005) Molecular electronics: Some views on transport junctions and beyond. Proc. Natl. Acad. Sci. U.S.A. 102(25):88018808

15. Lau CS, Mol JA, Warner JH, \& Briggs GAD (2014) Nanoscale control of graphene electrodes. Physical Chemistry Chemical Physics 16:20398-20401.

16. Nef C, et al. (2014) High-yield fabrication of nm-size gaps in monolayer CVD graphene. Nanoscale 6(13):7249-7254.

17. Börrnert F, et al. (2012) Lattice Expansion in Seamless Bilayer Graphene Constrictions at High Bias. Nano Lett. 12(9):4455-4459.

18. Loh KP, Bao Q, Ang PK, \& Yang J (2010) The chemistry of graphene. Journal of Materials Chemistry 20(12):2277-2289.

19. Vanin M, Gath J, Thygesen KS, \& Jacobsen KW (2010) First-principles calculations of graphene nanoribbons in gaseous environments: Structural and electronic properties. Phys. Rev. B. 82(19):195411.

20. Soler JM, et al. (2002) The SIESTA method for ab initio order- N materials simulation. Journal of Physics: Condensed Matter 14(11):2745.

21. Perdew JP, Burke K, \& Ernzerhof M (1996) Generalized Gradient Approximation Made Simple. Phys. Rev. Lett. 77(18):3865-3868.

22. Ferrer J, et al. (2014) GOLLUM: a next-generation simulation tool for electron, thermal and spin transport. New J Phys 16:093029. 